\title{
What's new for the clinician: excerpts from and summaries of recently published papers
}

SADJ June 2019, Vol. 74 No. 5 p255 - p258

Compiled and edited by $\mathrm{V}$ Yengopal

\section{Tooth crown discoloration induced by endodontic sealers: a 3-year evaluation}

\author{
MA Ekici, A Ekici, T Kaskat, et al. Clin Oral Invest. 2019; 23:2097.
}

https://doi.org/10.1007/s00784-018-2629-1.

\begin{abstract}
Discoloration of a single tooth is a demanding clinical issue especially if present in the aesthetic zone. It can be due to calcification of the pulp chamber, pulp necrosis and/or iatrogenic mishaps during various stages of endodontic treatment. Teeth often become discoloured consequent to the influence of endodontic materials, which can lead to an unpleasant appearance.
\end{abstract}

Tooth discoloration caused by restorative and endodontic materials has been evaluated with subjective approaches and objective methods. ${ }^{1}$ Spectrophotometers, colorimeters, digital photography, and digital image analysis techniques enable the objective evaluation of colour and colour change over time. ${ }^{1}$

Instrumental measurements commonly employ the Commission International de L'Eclairage's (CIE's) $L^{*}$, $a^{*}, b^{\star}$ system. The CIE L*a*b* colour coordinates enable the determination of colour in three-dimensional space.

$L^{*}$ values describe lightness, which ranges from black (0) to white (100); $a^{*}$ values represents red $\left(+80 a^{\star}\right)$ to green $\left(-80 a^{\star}\right)$ colour changes; and $b^{\star}$ values represent yellow $\left(+80 b^{\star}\right)$ to blue $\left(-80 b^{\star}\right)$ colour changes. ${ }^{1}$

Ekici and colleagues (2019) ${ }^{1}$ reported on a study that sought to evaluate the tooth crown discoloration induced by commonly used and new-generation endodontic sealers at one month and one and three years after application.

\section{METHODS}

Sixty human mandibular incisors that were free of caries, restorations, developmental defects, and crown discoloration were used.

Veerasamy Yengopal: $B C h D, B S c H o n s, M C h D, P H D$, Community Dentistry Department, School of Oral Health Sciences, University of Witwatersrand, Medical School, no. 7 York Road, Parktown 2193, South Africa. Email: veerasamy.yengopal@wits.ac.za
The teeth were cleaned using an ultrasonic method and a rubber cup to remove debris and extrinsic stains from the crown surfaces. The crowns were removed $2 \mathrm{~mm}$ below the cemento-enamel junction with highspeed diamond discs under water coolant.

The pulp was extirpated with a dental spoon, and the pulp chambers were chemo-mechanically debrided through the apical access. The prepared cavities were rinsed with $2 \%$ sodium hypochlorite, followed by sterile saline solution, and then dried with paper points and air-water spray.

The specimens were divided randomly into four groups $(n=15)$. The pulp chambers were filled with Pulpispad in group 1, AH26 in group 2, MTA Fillapex in group 3, and EndoREZ in group 4. The sealer compositions and manufacturer's information are presented in Table 1.

Sealers were prepared according to the manufacturers' instructions and left intentionally in the pulp chambers. After the specimens were sealed from the apical side using a composite resin (Charisma), they were placed into tap water-filled tubes, and then stored in an incubator (temperature, $37^{\circ} \mathrm{C}$; humidity, $100 \%$ ) until their respective evaluation times were reached.

The colour of the specimens was measured with a spectrophotometer (Vita Easyshade Compact) before sealer placement (baseline) and at one month and one and three years thereafter. For standardization, the same operator performed all colour evaluations. A mounting system was used for the customization and the reproducibility of the position of each crown. The cylindrical inner frame (diameter $=2 \mathrm{~cm}$, height $=0.2 \mathrm{~cm}$ ) of the blackbakelite sample assembly with which the spectrophotometer was equipped, was filled with black, nonpolychromatic, thermo-plasticized silicone.

All spectrophotometric measurements were performed under constant laboratory illumination in "tooth single" 


\begin{tabular}{|c|c|c|c|}
\hline Group & Material & Manufacturer & Composition according to manufacturers \\
\hline 1 & Pulpispad & Dentsply, France & $\begin{array}{c}\text { Silver powder, diiodothymol, zinc oxide, menthol, parachlorophenol, } \\
\text { synthetic camphor, eugenol. }\end{array}$ \\
\hline 2 & $\mathrm{AH} 26$ & $\begin{array}{l}\text { Dentsply DeTrey GmbH, } \\
\text { Konstanz, Germany }\end{array}$ & $\begin{array}{l}\text { Bismuth oxide, methenamine, titanium dioxide, silver phenol A, } \\
\text { epichlorohydrin resin, pigments. }\end{array}$ \\
\hline 3 & $\begin{array}{l}\text { MTA } \\
\text { Fillapex }\end{array}$ & Angelus, Londrina, Brazil & $\begin{array}{l}\text { Resins (salicylate, diluting, natural), bismuth trioxide, nanoparticulated silica, } \\
\text { mineral trioxide aggregate, pigments. }\end{array}$ \\
\hline 4 & EndoREZ & $\begin{array}{l}\text { Ultradent, South Jordan, } \\
\text { UT, USA }\end{array}$ & Urethane dimetacrylate resin matrix, zinc oxide, barium sulfate, pigments. \\
\hline
\end{tabular}

mode after spectrophotometer calibration. Colour measurement was repeated twice for each sample, and recorded using the $\mathrm{CIE} L^{*} a^{*} b^{*}$ system. The $L^{*} a^{*} b^{*}$ values were used to calculate changes in colour $(\Delta E)$ as follows:

$\triangle E L \star a * b *=$

$\left[(\Delta L)_{2}+(\Delta a)_{2}+(\Delta b)_{2}\right]{ }_{12} \Delta E L * a * b *=[(\Delta L) 2+(\Delta a) 2+(\Delta b) 2] 12$

$\Delta L *=L * 1--L * 0 \Delta L *=L * 1--L * 0$

$\Delta a * a * 1--a * 0 \Delta a * a * 1--a * 0$

$\Delta b * b * 1--b * 0 . \Delta b *=b * 1--b * 0$

$\Delta E, \Delta L, \Delta a$, and $\Delta b$ values thus represented differences from baseline at each timepoint.

The means of $\Delta E, \Delta L, \Delta a$, and $\Delta b$ values were calculated for each group and timepoint. The significance of changes over time was assessed using repeated-measures analysis of variance and Tukey's honestly significant difference test.

\section{RESULTS}

All sealers caused discoloration over time. Tooth colour change values were as follows: after one month (T1), group 1 = group $2>$ group 3 =group 4; after one year (T2), group 1 =group 2 =group $4>$ group 3 ; and after three years (T3), group $1>$ group $2=$ group $3=$ group $4(p<0.05)$.

So, groups 1 and 2 showed more colour change which differed from groups 3 and 4 at T1 $(p<0.05)$. At T2 (1 year), group 3 showed less colour change which differed from the other groups $(p<0.05)$. At T3 (3 years), group 1 showed more colour change which differed from the other groups $(p<0.05)$. Group 1 displayed an increase in mean $\Delta E$ values at time periods.

Within-group comparison for group 1 revealed a statistically difference between $\Delta E$ at $\mathrm{T} 1$ compared with $\Delta E$ at T2, T3 $(p<0.05)$. The $\Delta E$ value of group 2 at T3 was statistically less than $\Delta E$ value at other time periods $(p<0.05)$. Group 3 had the highest $\Delta E$ value at T3 and there was a statistically significant difference between $\Delta E$ at $\mathrm{T} 1$ and $\Delta E$ at T3 $(p<0.05)$. There was no statistically significant difference between the $\Delta \mathrm{E}$ values of group 4 at all time periods $(p>0.05)$.

The mean $\Delta L^{*}$ values of groups 1 and 2 were statistically lower than mean $\Delta L^{*}$ values of groups 3 and 4 at T1 $(p<0.05)$. There was a statistically significant difference between all groups at T2 $(p<0.05)$. At T3, the $\Delta L^{*}$ value of group 1 was statistically lower than $\Delta L^{*}$ value of other groups $(p<0.05)$. For group 1 , the mean $\Delta L^{*}$ value at T1 was statistically higher than mean $\Delta L^{*}$ values at T2 and T3 $(p<0.05)$. For group 2 , the mean $\Delta L^{*}$ value at T3 was statistically lower than mean $\Delta L^{*}$ values at T2 $(p<0.05)$. For group 3 , the mean $\Delta L^{*}$ value at T3 was statistically lower than mean $\Delta L^{*}$ values at $\mathrm{T} 1 \quad(p<0.05)$. For group 4, the mean $\Delta L^{*}$ value at T2 was statistically lower than mean $\Delta L^{*}$ values at T1 and T3 $(<0.05)$.

At $T 1$, the mean $\Delta a^{*}$ values of group 2 were statistically lower than other groups $(p<0.05)$. At $T 2$, the mean $\Delta a^{*}$ value of group 3 was statistically lower than other groups $(p<0.05)$. At T3, the mean $\Delta a^{*}$ values of group 1 were statistically higher than other groups $(p<0.05)$. For groups 1 and 2, there was a statistically significant difference between mean $\Delta a^{*}$ values at all time periods $(p<0.05)$. For group 3 , the mean $\Delta a^{*}$ value at T2 was statistically higher than mean $\Delta a^{*}$ values at T1 and T3 $(p<0.05)$. For group 4 , the mean $\Delta a^{*}$ value at $T 1$ was statistically lower than mean $\Delta a^{\star}$ values at T2 and T3 $(p<0.05)$.

At $T 1$, the mean $\Delta b^{*}$ value of group 2 was statistically lower than other groups $(p<0.05)$. At T2, the mean $\Delta b^{*}$ values of groups 2 and 4 were statistically higher than the mean $\Delta b^{*}$ values of groups 1 and $3(p<0.05)$.

At T3, the mean $\Delta b^{*}$ value of group 1 was statistically lower than the mean $\Delta b^{*}$ value of group $3(<0.05)$. For groups 1 and 3 , the mean $\Delta b^{\star}$ value at T3 was statistically higher than mean $\Delta b^{*}$ values at other time periods $(p<0.05)$. For groups 2 and 4 , there were statistically significant differences between $\Delta b^{*}$ values at all time periods $(p<0.05)$.

\section{CONCLUSIONS}

The researchers concluded that all tested sealers induced varying chromatic alterations at different time intervals.

\section{Implications for practice:}

$\Delta E$ value changes were correlated with changes in $\Delta L$, $\Delta a$, and $\Delta b$ values. Because the $\Delta E$ values of all tested sealers were sufficiently high so as to represent clinically visible colour changes, it is recommended that dentists remove residual sealer from treated pulp chambers thoroughly following obturation of root canals, before placing permanent restorations.

\section{Reference}

1. Ekici MA, Ekici A, Kaskat T, et al. Tooth crown discoloration induced by endodontic sealers: a 3-year ex vivo evaluation. Clin Oral Invest. 2019; 23:2097. https://doi.org/10.1007/s00784018-2629-1. 


\title{
2. Effect of air-blowing time and long-term storage on bond strength of universal adhesives to dentin
}

\author{
P Saikaew, J Fu, Chowdhury AFMA. Clin Oral Invest. 2019; 23:2629. \\ https://doi.org/10.1007/s00784-018-2656-y.
}

Direct tooth-coloured restoration has become routine for dental practice and attention has been given to improve effectiveness and longevity of resin-dentin bonds.

The newly introduced universal adhesive systems rapidly gained attention from dental clinicians due to their flexible application mode. ${ }^{1}$ Considering their compositions, universal adhesives are similar to the one-step self-etching system since all ingredients are incorporated into one bottle.

Air-drying after application of the adhesive is considered a crucial step in the bonding procedure. ${ }^{1}$ Within that step alone, many factors can affect the bond strength, including air-blowing time, air pressure, and air temperature. ${ }^{1}$ According to the conventional 3 -step etch and rinse adhesive systems, the air-blowing step is necessary to evaporate the solvent from the primer and to achieve a uniform, thin bonding layer.

In general, the recommended air-blowing duration for the newest universal adhesives range from $5 \mathrm{~s}$ to $15 \mathrm{~s}$, or until the liquid film on the surface is no longer visibly mobile, but the specific air-blowing time is not standard. Inadequate air-blowing can lead to insufficient solvent evaporation, and therefore, the residual solvent can affect the mechanical properties of the adhesives and the bond strength. ${ }^{1}$

Indeed, extended air-blowing time was reported to increase the bond strength by improving solvent evaporation. However, the effect appears to be solvent-dependent. Hence, the effect of air-blowing time on the resin-dentin bond is still unclear.

Saikaew and colleagues (2019) ${ }^{1}$ undertook a study that sought to evaluate the effect of air-blowing time on the microtensile bond strength ( $\mu \mathrm{TBS}$ ) of three universal adhesives to dentin after $24 \mathrm{~h}$ and one year. The null hypothesis tested was that the airblowing time had no immediate or long-term effect on the resin-dentin bond strength of universal adhesives.

\section{METHODS}

Ninety extracted human third molars were used in this study. The teeth were free from decay and cracks. They were stored in an aqueous solution of $0.5 \%$ chloramine-T at $4^{\circ} \mathrm{C}$ and used within six months of storage.

The mid-coronal dentin surface was exposed by grinding the occlusal enamel with a gypsum model trimmer. The flat surfaces were confirmed by using a light microscope that no enamel remained. After that, the teeth were ground manually with 600-grit $\mathrm{SiC}$ paper under running water for one min.

Five teeth per group were randomly assigned into 18 experimental groups according to three adhesives, three air-blowing durations, and two storage times. The adhesives used in this study were Clearfil Universal Bond, G-Premio Bond, and Scotchbond Universal Adhesive. Details of adhesive applications and compositions are listed in Table 1.

The adhesive application was performed according to the manufacturer's instruction. After adhesive application, each group was assigned for $5 \mathrm{~s}, 15 \mathrm{~s}$, or $30 \mathrm{~s}$ of air-blowing time and then light-cured at $>1200 \mathrm{~mW} / \mathrm{cm}^{2}$ for 10s. All bonded surfaces were restored with two layers of $2 \mathrm{~mm}$-thick resin composite (Clearfil AP-X). Each layer was light-cured for 20 s.

After storage in distilled water at $37^{\circ} \mathrm{C}$ for $24 \mathrm{~h}$, each bonded tooth was sectioned into slabs using Isomet diamond saw. Three resin-dentin slabs from the central area were selected and further cut into beams (crosssectional area approximately $1 \mathrm{~mm}^{2}$ ) and resin-dentin slabs from the crown periphery were separated for observation of the resin-dentin interface.

For $\mu$ TBS test, resin-dentin beams were then subjected to $\mu$ TBS test for immediate bond strength or tested after one year of water storage for the long-term test. Fifteen randomly selected beams originated from five

\begin{tabular}{|c|c|c|c|}
\hline Adhesive & $\mathrm{pH}$ & Composition & Manufacturers' instruction \\
\hline $\begin{array}{c}\text { Clearfil } \\
\text { Universal Bond }\end{array}$ & 2.3 & $\begin{array}{l}\text { 10-MDP, Bis-GMA, HEMA, } \\
\text { ethanol, hydrophilic aliphatic } \\
\text { dimethacrylate, colloidal silica, } \\
\text { dl-camphorquinone, silane } \\
\text { coupling agent, and water. }\end{array}$ & $\begin{array}{l}\text { 1. Apply the adhesive to the dentin surface with applicator brush and rub it for } 10 \text { s. } \\
\text { 2. Dry the dentin surface sufficiently by blowing mild air for more than } 5 \text { s until the adhesive } \\
\text { does not move. } \\
\text { 3. Light cure for } 10 \text { s. }\end{array}$ \\
\hline G-Premio Bond & 1.5 & $\begin{array}{l}\text { 10-MDP, } 4 \text { methacryloxyethyl- } \\
\text { trimellitate anhydrate, dimethac- } \\
\text { rylate monomer, distilled water, } \\
\text { acetone, photo initiators, and } \\
\text { silica fine powder. }\end{array}$ & $\begin{array}{l}\text { 1. Apply using a micro-brush. } \\
\text { 2. Leave undisturbed for } 10 \text { s after application. } \\
\text { 3. Dry thoroughly for } 5 \mathrm{~s} \text { with oil free air under maximum air pressure. } \\
\text { 4. Light cure for } 10 \mathrm{~s} \text {. }\end{array}$ \\
\hline $\begin{array}{l}\text { Scotchbond } \\
\text { Universal }\end{array}$ & 2.7 & $\begin{array}{l}\text { 10-MDP, HEMA, silane, dimeth- } \\
\text { acrylate resins, Vitrebond }{ }^{\mathrm{TM}} \\
\text { copolymer, filler, ethanol, water, } \\
\text { and initiators. }\end{array}$ & $\begin{array}{l}\text { 1. Apply the adhesive on the surface and rub it for } 20 \text { s. } \\
\text { 2. Gently air-dry the adhesive for approximately } 5 \text { s for the solvent to evaporate. } \\
\text { 3. Light cure for } 10 \text { s }\end{array}$ \\
\hline
\end{tabular}


teeth were tested per group. A cyanoacrylate glue was used to attach the beam to a Ciucchi's jig. A tensile force was applied at a crosshead speed of $1 \mathrm{~mm} / \mathrm{min}$ in a desktop testing apparatus until failure occurred. The beams that failed involving the glue or failed at the glue before beam fracture were excluded from the analysis. $\mu \mathrm{TBS}$ was expressed in MPa by the division of applied force $(\mathrm{N})$ at the time of fracture by the bonded area $\left(\mathrm{mm}^{2}\right)$. The data were then analysed by three-way ANOVA and Duncan test $(\alpha=0.05)$.

The fractured specimens were carefully removed from the jig and mounted on an aluminum stub. The overall failure mode was determined using SEM at an accelerating voltage of $10 \mathrm{kV}$ with low magnification $(\times 80)$. Failure mode categories were classified into cohesive in composite, cohesive in dentin, cohesive in the bonding agent, adhesive at interface, and mixed involving interfacial de-bonding. The specific features of fracture surfaces were further observed at high magnification by SEM $(\times 10,000)$.

Two resin-dentin slabs from the peripheral part of the tooth prepared for $\mu$ TBS were used for resin-dentin interface observation. The internal surface of the slab was polished with a series of $\mathrm{SiC}$ papers under running water and diamond pastes. After that, the surface was treated with $5 \% \mathrm{HCl}$ for $30 \mathrm{~s}$ followed by $\mathrm{NaOCl}$ for $5 \mathrm{~min}$. The slabs were left dried overnight in an incubator. Finally, they were sputter-coated and examined using SEM at $\times 3000$ magnification .

\section{RESULTS}

Microtensile bond strength ( $\mu \mathrm{TBS}$ ): There were no pretest failures observed in this study. Three-way ANOVA revealed a significant effect of adhesive $(p<0.0001)$, air-blowing time $(p=0.002)$, and storage time $(p<0.0001)$. The interaction between the three variables was also significant $(p=0.026)$.

As for the immediate bond strength, highest bond strength was observed with Scotchbond. There was no effect of air-blowing time on the immediate bond strength of Scotchbond and G-Premio Bond whereas increasing air-blowing time to 30s for Clearfil Universal Bond resulted in statistically significant higher bond strength than that of 5 s air-blowing time.

Long-term bond strengths of all groups were significantly lower than those of immediate bond strength, except for Clearfil Universal Bond at $5 \mathrm{~s}$ and 30 s of airblowing time. When compared with $5 \mathrm{~s}$ of air-blowing time, the extension of air-blowing time to $15 \mathrm{~s}$ and $30 \mathrm{~s}$ led to significantly higher long-term bond strength with Scotchbond and Clearfil Universal Bond, respectively, while air-blowing time showed no effect on G-Premio Bond.

\section{Failure analysis}

For Scotchbond, the failure mode was mainly categorized into cohesive in dentin. On the other hand, adhesive failure at interface and cohesive in bonding were the mainly observed failure modes in Clearfil Universal bond and G-Premio Bond except for Clearfil Universal Bond at $15 \mathrm{~s}$ blowing time which showed the highest percentage of cohesive failures in dentin at both test periods.

Resin-dentin interface observation: The thicknesses of the adhesive layers were between two and $10 \mu \mathrm{m}$. Thinner adhesive layers were noticed after extended air-blowing time groups compared with those of $5 \mathrm{~s}$. Micron-sized voids were detected in the adhesive layers of G-Premio Bond, which were approximately $2-5 \mu \mathrm{m}$ in diameter. A lesser number of voids was observed with increased air-blowing time.

\section{CONCLUSIONS}

The researchers concluded that air-blowing time had no influence on the bond strength of the acetone-based adhesive (G-Premio Bond). On the other hand, extension of air-blowing time tended to have a beneficial effect on the ethanol-based adhesives (Clearfil \& Scotchbond).

Long-term bond strength of Clearfil \& Scotchbond were significantly increased, whereas improvement of immediate bond strength was only found in Clearfil.

\section{Implications for practice}

These results suggest that extended airblowing times can only increase bond strengths in general for Clearfil \& Scotchbond.

\section{Reference}

1. Saikaew P, Fu J, Chowdhury AFMA. Effect of air-blowing time and long-term storage on bond strength of universal adhesives to dentin. Clin Oral Invest. 2019; 23:2629. https:// doi.org/10.1007/s00784-018-2656-y. 